\title{
First results from two deep Askaryan Radio Array stations
}

Thomas Meures* for the ARA Collaboration

University of Wisconsin

E-mail: meureseicecube.wisc.edu

The Askaryan Radio Arrray (ARA) is a planned and in parts constructed detector for ultra-high energy neutrinos, utilizing radio emission from neutrino induced particle showers. With this detection method, ARA will be able to employ several gigatons of South-Pole ice as a detector medium needed to efficiently discover neutrinos with energies above $10 \mathrm{PeV}$. These neutrinos carry particularly interesting information about highest energy processes in the far universe. The detector is planned to consist of 37 widely-separated antenna clusters, so-called stations. Currently, 3 stations are deployed in the ice recording transient radio waves, with two more stations assembled for deployment in the austral summer 2017/2018. In this presentation, first analysis results from data recorded by two ARA stations in the year 2013 are summarized.

38th International Conference on High Energy Physics 3-10 August 2016

Chicago, USA

${ }^{*}$ Speaker. 


\section{Introduction}

The Askaryan Radio Array (ARA) is a detector under construction, dedicated to the search of ultra-high energy neutrinos. It is optimized in particular for neutrinos produced in the GZK mechanism, a phenomenon predicted by Greisen, Zatsepin and Kuzmin in 1966 [1, 2]. At cosmic ray energies beyond $10^{19.5} \mathrm{eV}$, the cross section for pion production in the interaction between a cosmic ray particle and the omnipresent microwave background has a resonance. The such generated pions decay into, amongst others, neutrinos [3]. On these premises it can be expected that cosmic ray particles of such energies or higher are mostly absorbed within $100 \mathrm{Mpc}$ and a flux of so-called cosmogenic neutrinos is generated. The magnitude and energy distribution of this flux depends mainly on the source distribution, the mass and the spectrum of ultra-high energy cosmic rays. Hence, a measurement of the cosmogenic neutrino flux can help to constrain the above mentioned properties of ultra-high energy cosmic rays.

The expected number of cosmogenic neutrinos is estimated to produce less than 1 interaction in $1 \mathrm{~km}^{3}$ of water equivalent per year. At such low flux intensities neutrino detection is not efficient via optical sensors but can be achieved by utilizing radio emission via the Askaryan effect. As described in $[4,5]$, particle cascades produced by the interaction of ultra-high energy neutrinos are predicted to build up a net negative charge of some $10 \%$ which, as it is moving, emits electromagnetic radiation. This radiation becomes coherent for wavelengths comparable to the lateral cascade dimensions, hence, in the radio regime. The above effect has been verified in beam tests for various materials including ice [6]. In radio-transparent media like South Pole ice, radio waves have an attenuation length of more than $800 \mathrm{~m}$ which allows for the survey of a large volume with a relatively small number of sensors and thus for the cost effective construction of a neutrino detector [7].

\section{The detector}

The baseline design of the ARA detector, shown in Figure 1, foresees the deployment of 37 antenna clusters in the South Pole ice. With a thickness of $3000 \mathrm{~m}$, a vast area and its remote location relative to populated areas, the South Pole ice sheet provides a very good environment for a radio detector. The antenna clusters, in the following referred to as "stations", consist each of 16 measurement antennas which form a cube of $20 \mathrm{~m}$ at each side. The antennas are deployed into holes at a depth of roughly $180 \mathrm{~m}$. Such a deep deployment is favorable because the index of refraction in the upper $200 \mathrm{~m}$ of the South Pole ice sheet changes with depth [8]. This causes radio rays to be bend downwards and prevents them from reaching shallow regions of the ice. Hence, the sensitivity of a radio detector depends on the antenna depth within the top $200 \mathrm{~m}$ of the ice, favoring a deep deployment.

The used antennas are sensitive to a single polarization of radio signals, half of them to vertical (Vpol) and the other half to horizontal polarization (Hpol). They operate in a frequency range between 150 and $850 \mathrm{MHz}$. Around the measurement antennas, four calibration pulsers, two of each polarization, are distributed in the ice. These are used for timing and geometry calibration of the measurement cluster. The signal from the measurement antennas is filtered and amplified with a low noise amplifier close to the antenna, before being transmitted to the surface via an analog fiber 

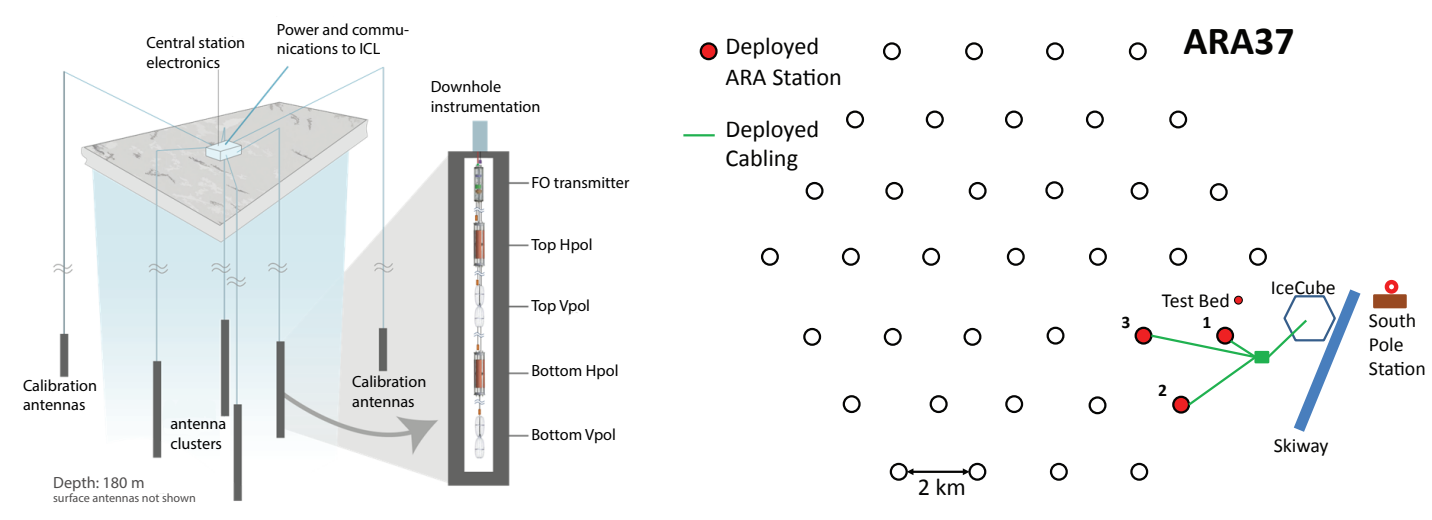

Figure 1: Left: A sketch of one ARA station with zoom into the in-ice measurement system. Right: The full ARA detector as planned to be contruccted at South Pole, depicting the currently installed stations in red.

link. In this way, noise pickup and signal loss through elongated copper cables can be avoided. At the surface of the ice, the signal is split to be fed into a trigger- and a digitizer-system. Both systems are controlled by a Field Programmable Gate Array (FPGA) in combination with a Single Board Computer (SBC). Whenever the signal on 3 out of 8 antennas of one polarization crosses a given trigger threshold, digitization is initialized and the recorded data is sent to storage as an ARA-event. The system noise, including the ice and other ambient sources, has been determined to correspond to roughly $350 \mathrm{~K}$ in the relevant frequency range. Trigger thresholds are tuned to generate station triggers at a rate of $5 \mathrm{~Hz}$. This can be achieved with an average voltage threshold of 2.5 times the noise RMS on each channel.

Each ARA station functions as an autonomous neutrino detector. To minimize overlap between stations and, thus, maximize the effective area of the full detector the stations are distributed in a hexagonal structure with a spacing of $2 \mathrm{~km}$. More details about the ARA stations and their performance can be found in [7,9].

Currently, 3 ARA stations and a prototype are deployed at the South Pole. The prototype station, called Testbed, has been used to determine ice properties for radio measurements and for an initial neutrino search, described in [10]. It was decommissioned in 2013.

\section{Latest data analysis results from two ARA stations}

The latest results from an analysis of ARA data are based on the 10 first months of operation of two ARA stations in 2013. During this time the two stations ran with an uptime of roughly $75 \%$. The uptime has been significantly improved by now, thanks to better monitoring algorithms and partly automated recovery systems. In the following, a summary of the analysis will be presented. More details can be found in [9].

Simulated neutrino events, used to train analysis algorithms and to calculate the detector effective area and sensitivity, are generated with the AraSim code [11]. In this simulation, interactions of neutrinos with energies in the range between $10^{16}$ and $10^{21} \mathrm{eV}$ are generated with a random vertex position around the station and a random incoming direction of the neutrino. The estimated signal emission is propagated to the ARA antennas, taking into account the depth dependent atten- 

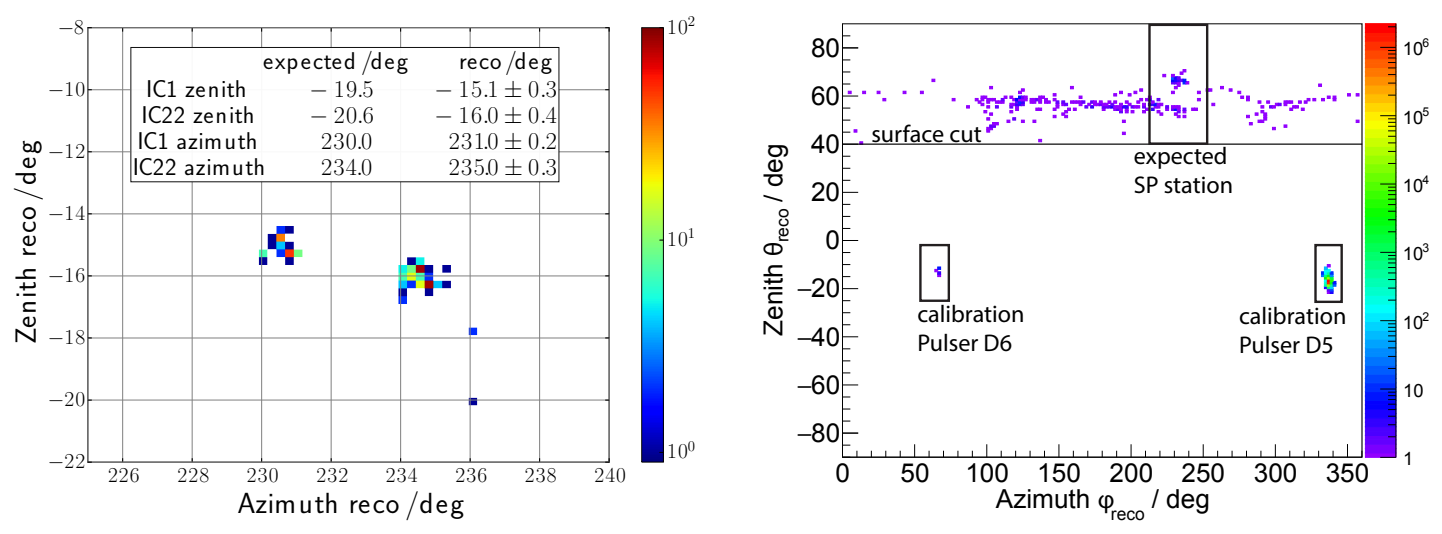

Figure 2: Left: The reconstruction after analysis cuts of pulser signals from two sources deployed with the last IceCube strings. The true and the reconstructed angles are shown in the legend. Right: The Skymap of reconstructible impulsive events in station ARA3. Angular cuts are indicated by black lines. No event has been found outside the cut regions.

uation length and the changing index of refraction in the ice. The antenna gain of ARA antennas is estimated from a combination of measurement and simulation results. The thermal background noise is estimated from the ice temperature profile and measurements of cosmic radio sources. The signal chain noise figure has been measured at room temperature and is extrapolated to the colder environment. More details about the detector calibration can be found in [9].

The data analysis is performed in two main steps. Since most recorded data is thermal noise, first, a simple filter is used to distinguish impulsive radio events from the thermal background. This can be achieved by producing hit timing patterns and by weighting the number of hits with a measure of conformity of their distribution to a passing wavefront. This procedure reduces the amount of data by roughly 10 orders of magnitude, while retaining $60 \%$ of simulated triggering neutrino events with a primary energy of $10^{18} \mathrm{eV}$. In the next step, the reduced set of ARA-events is analyzed with an angular vertex reconstruction algorithm. This reconstruction is based on the arrival time difference between signals on different antennas, calculated via cross-correlation. Apart from determining the incoming direction of radio signals in an event the algorithm further filters impulsive events with non-causal hit patterns. The reconstructed events can then be separated into background and neutrino events by angular cuts. The South Pole ice sheet acts as a filter, not allowing for other particles than neutrinos to generate visible impulsive radio signals in the deep ice. Therefore, potential neutrino candidates are identified by excluding all events with vertex reconstruction pointing to the ice surface or known radio sources in the ice.

Figure 2 illustrates the reconstruction precision and the applied angular cuts for one of the ARA stations. Since the reconstruction does not account for the changing index of refraction in the ice, the zenith angle reconstruction is offset by a few degree from the true value. Apart from that, reconstruction errors generally amount to less than $2^{\circ}$.

Using the above described simulation and a flux prediction from [17], an expectation of 0.11 neutrinos can be estimated to survive all analysis cuts within the given lifetime. In agreement to this, no neutrino candidate has been found after angular cuts. In absence of a discovery a limit can be 
calculated as shown in Figure 3 in comparison to selected flux predictions and other detector limits. It incorporates systematic errors which are dominantly related to the signal chain calibration. The limit is not competitive to the current best neutrino detectors in the regarded energy range but it suggests the full array will have access to leading neutrino flux models at energies of $100 \mathrm{PeV}$ and above.

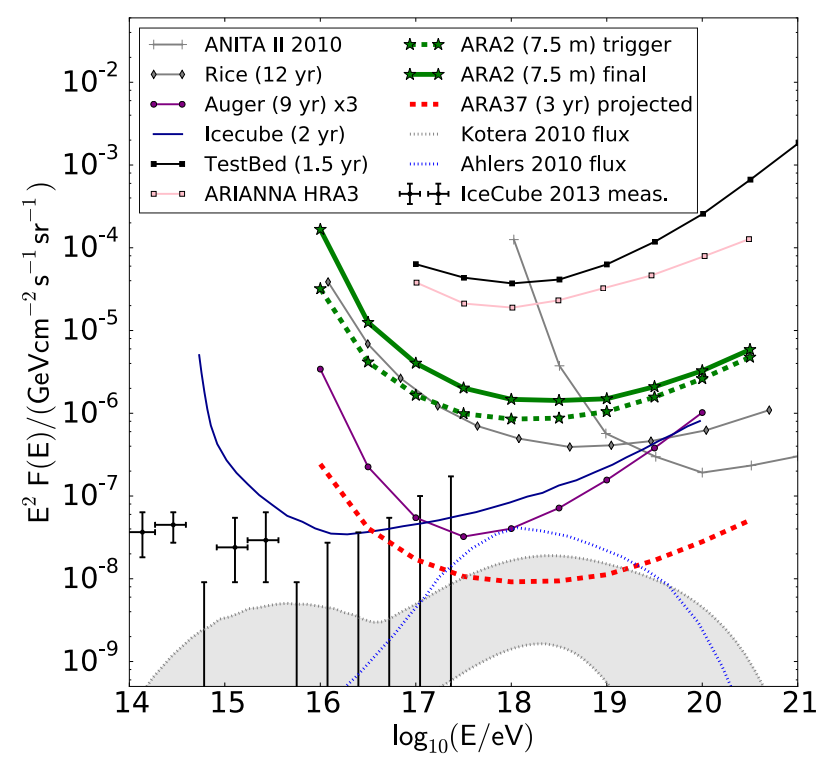

Figure 3: The ARA 2 station neutrino limit for 7.5 months of operation compared to other detector limits and flux expectations. The projection for the ARA37 sensitivity is calculated at trigger level without accounting for systematic errors $[9,10,13,14,12,15,16,17,18]$.

\section{Future activities}

Currently two more ARA stations, ARA4 and ARA5 are being prepared for deployment in the ice during the 2017/2018 South Pole season. As coincident events between stations are rare by design, the effective area of the ARA detector scales close to the number of stations. Hence, the 2018 detector will be at least twice as sensitive as the detector has been in 2013 and is expected to run at a higher uptime percentage.

Furthermore, research is underway to reduce the minimal trigger threshold by using a phased array of antennas. By adding the signal waveform on several antennas, random noise can be reduced by a factor of $\sqrt{n}$ versus impulsive signals, with $n$ the number of antennas, when the signals perfectly overlap. This perfect overlap can be found by adding antenna signals with all possible phase shift combinations and selecting the combination with maximum signal outcome. A detailed description of this method and simulation results can be found in [19]. For ARA a system of 16 antennas, mounted on one vertical string, is currently being integrated. The antenna signals are digitally combined with variable phase shift combinations and the maximum signal peak is used to trigger. In this way the trigger sensitivity of an ARA station could be improved by up to a factor of 4 . Consequently, the energy threshold of ARA would drop and render the ARA37 detector competitive with current experiments in the low PeV energy range. 


\section{References}

[1] K. Greisen, End to the Cosmic-Ray Spectrum?, Phys. Rev. Lett. 16 (748) 1966

[2] G. T. Zatsepin, V. A. Kuzmin, Upper limit of the spectrum of cosmic rays, JETP Lett. 4 (78) 1966

[3] V. S. Berezinsky, G. T. Zatsepin, Cosmic rays at ultrahigh-energies (neutrino?), Phys. Lett. B28 (423) 1969

[4] G. A. Askaryan, Excess negative charge of an electron-photon shower and its coherent radio emission, JETP 41 (616) 1962

[5] G. A. Askaryan, Coherent radio emission from cosmic showers in air and in dense media, JETP 48 (988) 1965

[6] P. W. Gorham et al., Observations of the Askaryan Effect in Ice, Phys. Rev. Lett. 99 (171101) 2007

[7] P. Allison et al. (ARA), Design and initial performance of the Askaryan Radio Array prototype EeV neutrino detector at the South Pole, Astroparticle Physics 35 (457) 2012

[8] I. Kravchenko, D. Besson, J. Meyers, In situ index-of-refraction measurements of the South Polar firn with the RICE detector, Journal of Glaciology 50 (522) 2004,

[9] P. Allison et al. (The ARA collaboration), Performance of two Askaryan Radio Array stations and first results in the search for ultrahigh energy neutrinos, Phys. Rev. D 93 (082003) 2016,

[arXiv:1507.08991]

[10] P. Allison et al. (The ARA collaboration), First Constraints on the Ultra-High Energy Neutrino Flux from a Prototype Station of the Askaryan Radio Array, Astropart. Phys. 70 (62) 2015, [arXiv:1404.5285]

[11] E. S. Hong, A. Connolly, C. G. Pfendner for the ARA Collaboration, Simulation of the ARA Experiment for the Detection of Ultrahigh Energy Neutrinos, in proceedings of 33rd International Cosmic Ray Conference (ICRC 2013), Rio de Janeiro, Brazil

[12] I. Kravchenko et al., Updated results from the RICE experiment and future prospects for ultra-high energy neutrino detection at the south pole, Phys. Rev. D 85 (062004) 2012

[13] M. G. Aartsen et al., Probing the origin of cosmic-rays with extremely high energy neutrinos using the IceCube Observatory, Phys.Rev. D88 (112008) 2013, [arXiv: 1310.5477 ],

[14] P. W. Gorham et al., Observational constraints on the ultrahigh energy cosmic neutrino flux from the second flight of the ANITA experiment, Phys. Rev. D82 (022004) 2010

[15] A. Aab et al., An improved limit to the diffuse flux of ultra-high energy neutrinos from the Pierre Auger Observatory, Phys. Rev. D91 (092008) 2015, [arXiv: 1504 . 05397]

[16] M. G. Aartsen et al. (IceCube), Observation of High-Energy Astrophysical Neutrinos in Three Years of IceCube Data, Phys. Rev. Lett. 113 (101101) 2014 [arXiv: 1405.5303 ]

[17] M. Ahlers et al., GZK neutrinos after the Fermi-LAT diffuse photon flux measurement, Astroparticle Physics 34 (106) 2010

[18] K. Kotera, Kumiko et al., The Astrophysics of Ultrahigh-Energy Cosmic Rays, Annual Review of Astronomy and Astrophysics 49 (119) 2011

[19] A. G. Vieregg, K. Bechtol, A. Romero-Wolf, A Technique for Detection of PeV Neutrinos Using a Phased Radio Array JCAP 2 (005) 2016, [arXiv: 1504 . 08006 ] 